\title{
METHOD OF DIMENSIONALITY REDUCTION IN CONTACT MECHANICS AND FRICTION: A USER'S HANDBOOK. II. POWER-LAW GRADED MATERIALS
}

\author{
UDC 539.3
}

\author{
Markus Hess, Valentin L. Popov \\ Department of System Dynamics and the Physics of Friction, TU Berlin, Germany
}

\begin{abstract}
Until recently, the only way of solving contact problems was to apply threedimensional contact theories. However, this presupposes higher mathematical and numerical knowledge, which usually only research groups possess. This has changed drastically with the development of the method of dimensionality reduction (MDR), which allows every practically oriented engineer an access to the solution of contact problems. The simple and contact-type dependent rules are summarized in the first part of the user manual; they require contacts between elastically homogeneous materials. The present paper forms the second part of the user handbook and is dedicated to the solution of contact problems between power-law graded materials. All the MDR-rules are listed with which normal, tangential and adhesive contacts between such highperformance materials can be calculated in a simple manner.
\end{abstract}

Key Words: Normal Contact, Tangential Contact, Adhesion, Power-law Graded Materials, Partial Slip, Method of Dimensionality Reduction

\section{INTRODUCTION}

The classical dimensionality reduction method is designed to solve contact problems between elastically homogeneous materials. Although it does not appear at first sight, the MDR unites all three-dimensional contact theories and transforms them in such a way that only simple rules remain which have to be applied to equivalent, one-dimensional contact problems [1]. These rules are summarized in the first part of the user handbook [2], assuming axisymmetric profiles and compact contact areas. However, Argatov et al. [3] showed that the MDR is also valid for arbitrarily shaped and non-compact contact areas.

Received October 24, 2016 / Accepted November 29, 2016

Corresponding author: Markus Hess

Institute of Mechanics, Berlin Institute of Technology, Strasse des 17. Juni 135, 10623 Berlin, Germany

E-mail: markus.hess@tu-berlin.de 
The enormous technological progress in recent times is closely linked to the development of high-performance materials. In order to meet the increased demands, functionally graded materials (FGM) are used, which include the elastically power-law graded materials. They are characterized by a modulus of elasticity which increases perpendicularly to the half-space surface according to the power law:

$$
E(z)=E_{0}\left(\frac{z}{c_{0}}\right)^{k} \text { with } \quad 0 \leq k<1 .
$$

where $c_{0}$ denotes the characteristic depth in which elastic modulus $E_{0}$ prevails independently of the exponent of the elastic inhomogeneity (see Fig. 1).
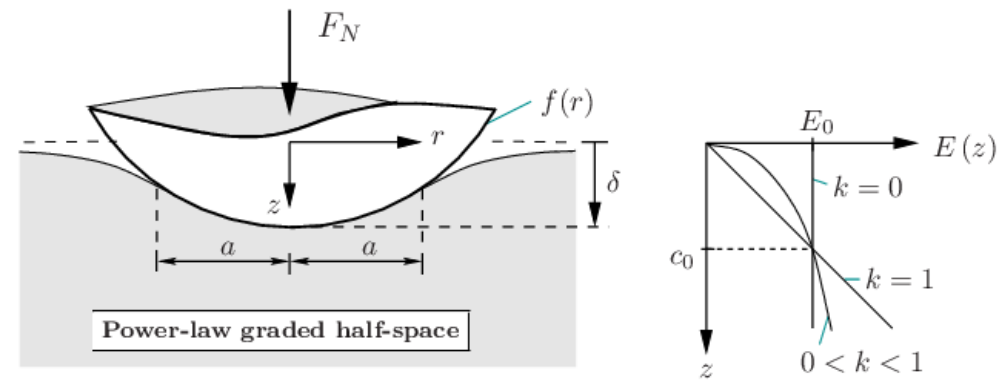

Fig. 1 Axisymmetric contact between a rigid indenter and an elastically power-law graded half-space

Contact mechanics of such materials were mainly developed by Booker et al. [4, 5] and Giannakopoulos and Suresh [6] for normal contacts without adhesion and Chen et al. [7] and Jin et al. [8] for adhesive contacts. Due to the interest in investigating the behavior of elastically inhomogeneous, biological structures as well as the adhesive material behavior in micro- and nanosystem technology the latter is still a subject of current research. An analytical solution of the tangential contact has not yet been published, but Hess [9] presented the solution at a recent workshop. The basic ideas were also mentioned in a further conference paper [10]. The key to the solution of the tangential contact lies once more in the superposition principle of Ciavarella [11] and Jäger [12]. Analogously to contact mechanics of homogeneous materials, all the above-mentioned contact theories for the calculation of contacts between power-law graded materials can again be suitably transformed by means of MDR, so that equivalent one-dimensional models are created which satisfy simple rules. The general foundations for the mapping of contacts between heterogeneous materials were given by Popov [13]. The derivation of all MDR-rules for the exact mapping of non-adhesive and adhesive normal contacts between power-law graded materials goes back to Hess $[14,15]$. In this paper, all the rules are listed and their easy handling for the solution of normal, tangential and adhesive contacts is explained by means of examples. 


\section{TWO INTRODUCTORY STEPS OF THE MDR}

The basic procedure for solving contact problems by MDR is independent of whether we consider homogeneous or inhomogeneous materials. Only the rules look a bit different. Again, we would like to assume axisymmetric contacts. Furthermore, the exponent of elastic inhomogeneity $k$ and characteristic depth $c_{0}$ of the contacting bodies should be the same. The two solids should thus be able to distinguish themselves only in the Poisson's ratios $v_{1}, v_{2}$ and / or in the moduli of elasticity $E_{01}, E_{02}$ prevailing in the characteristic depth.

\subsection{The first step: Mapping of material properties}

The power-law graded properties of the contacting bodies are taken into account within the MDR by linear elastic springs of suitable stiffness. In addition to a normal stiffness, each spring also has an independent tangential stiffness. The spring stiffnesses related to the distance of springs $\Delta x$ are called foundation moduli $c_{N}$, respectively $c_{T}$. These have to be chosen as follows:

$$
\begin{gathered}
c_{N}(x)=\left(\frac{1-v_{1}^{2}}{h_{N}\left(k, v_{1}\right) E_{01}}+\frac{1-v_{2}^{2}}{h_{N}\left(k, v_{2}\right) E_{02}}\right)^{-1}\left(\frac{|x|}{c_{0}}\right)^{k}, \\
c_{T}(x)=\left(\frac{1}{h_{T}\left(k, v_{1}\right) E_{01}}+\frac{1}{h_{T}\left(k, v_{2}\right) E_{02}}\right)^{-1}\left(\frac{|x|}{c_{0}}\right)^{k} .
\end{gathered}
$$

Coefficients $h_{N}$ and $h_{T}$ in the foundation moduli according to Eqs. (2) and (3) are complicated but well-defined functions depending on Poisson's ratio $v$ and exponent $k$ of the elastic inhomogeneity. They are given in the appendix. The decisive factor at the foundation moduli is that they depend on coordinate $x$ (see Fig. 2). Both stiffnesses increase with the lateral distance from the center point of the contact to exactly the same power law according to which, in the original problem, the elastic modulus increases perpendicularly to the half-space surface. In the special case of homogeneous half-space $k=0$, the following holds:

$$
h_{N}\left(0, v_{i}\right)=1 \quad \text { and } \quad h_{T}\left(0, v_{i}\right)=\frac{2}{\left(1+v_{i}\right)\left(2-v_{i}\right)} .
$$

Then coefficients $c_{N}$ and $c_{T}$ are constant and equal to effective elastic moduli $E^{*}$ and $G^{*}$ [2].

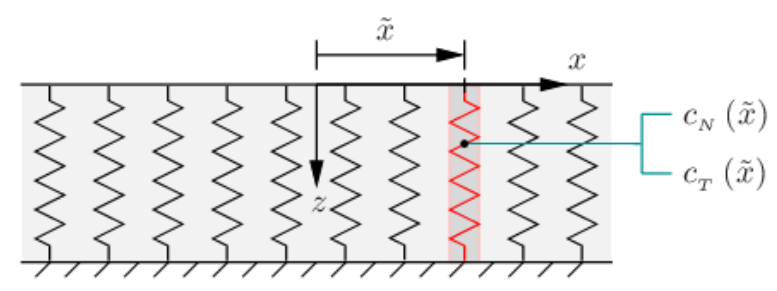

Fig. 2 Series of infinitesimaly adjacent, linear spring elements whose normal and tangential stiffness increase with the lateral distance from the midpoint of contact 


\subsection{The second step: Transformation of profile}

The second preliminary step involves the transformation of given three-dimensional contact profile $f(r)$ into an equivalent plane profile $g(x)$. The transformation and reverse transformation for the profile functions are [14]:

$$
\begin{gathered}
g(x)=|x|^{1-k} \int_{0}^{|x|} \frac{f^{\prime}(r)}{\left(x^{2}-r^{2}\right)^{\frac{1-k}{2}}} \mathrm{~d} r, \\
f(r)=\frac{2 \cos \left(\frac{k \pi}{2}\right)}{\pi} \int_{0}^{r} \frac{x^{k} g(x)}{\left(r^{2}-x^{2}\right)^{\frac{1+k}{2}}} \mathrm{~d} x .
\end{gathered}
$$

For better understanding, Fig. 3 visualizes the transformation of the profile. It should be noted that the equivalent plane profile is sometimes called equivalent $1 \mathrm{D}$ profile since it belongs to the equivalent 1D system.

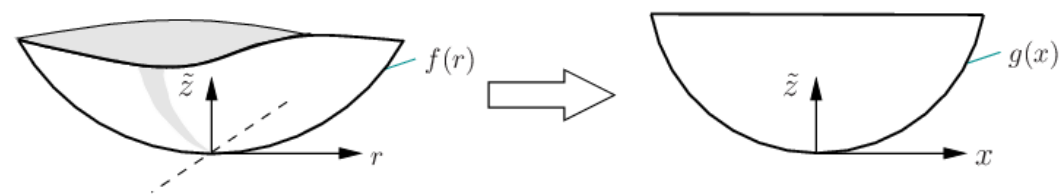

Fig. 3 Tranformation of the 3D-profile into an equivalent plane profile

\subsection{Example for the MDR transformation}

As an example we consider the transformation of a profile whose shape is described by the power function:

$$
f(r)=A_{n} r^{n} \text { with } n \in \mathbb{R}^{+}, A_{n}=\text { const . }
$$

Application of Eq. (5) to the profile according to Eq. (7) leads to the following equivalent profile:

$$
g(x)=\kappa(n, k) A_{n}|x|^{n}=\kappa(n, k) f(|x|),
$$

with

$$
\kappa(n, k)=\int_{0}^{1} \frac{n \varsigma^{n-1}}{\left(1-\varsigma^{2}\right)^{\frac{1-k}{2}}} d \varsigma=\frac{n}{2} \int_{0}^{1} t^{\frac{n}{2}-1}(1-t)^{\frac{k+1}{2}-1} \mathrm{~d} t=: \frac{n}{2} \mathrm{~B}\left(\frac{n}{2}, \frac{k+1}{2}\right),
$$

where $\mathrm{B}(x, y)$ is the complete beta function. Eq. (8) clearly indicates that the equivalent profile results from a simple, vertical scaling of the original profile. The scaling factor $\kappa(n, k)$ is dependent on the exponent of the power function and the exponent of the elastic inhomogeneity. The scaling factor increases with increasing exponent of the power-law profile (see Fig. 4). 


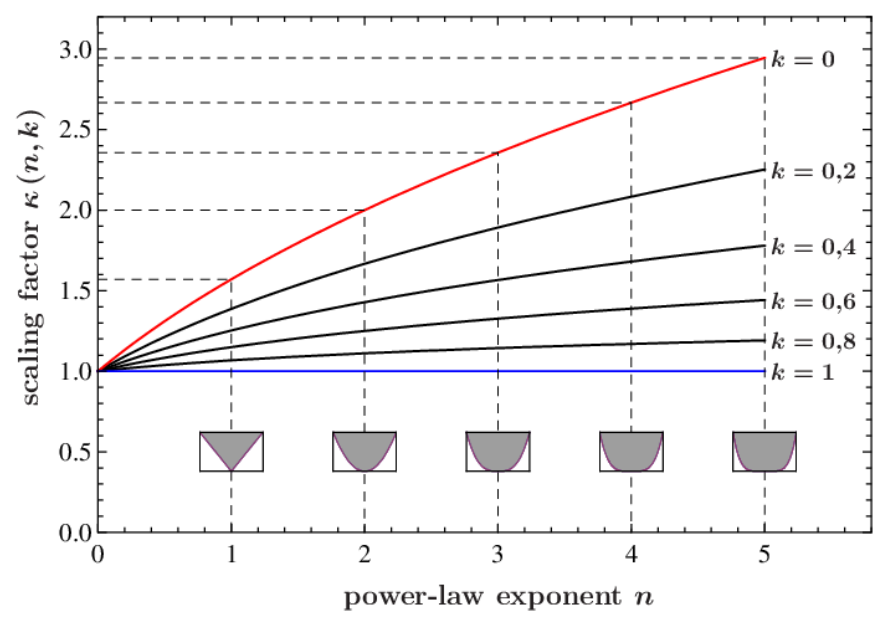

Fig. 4 Dependence of scaling factor $\kappa$ on power-law exponent $n$ for different exponents $k$ of the power-law graded material (adopted from [14])

In the homogeneous case, the known values $\kappa(1,0)=\pi / 2$ for the conical and $\kappa(2,0)=2$ for the parabolic indenter are obtained. The equivalent one-dimensional profiles of the basic contact profiles are listed in Table 1.

Table 1 Basic three-dimensional profiles and their equivalent one-dimensional profiles

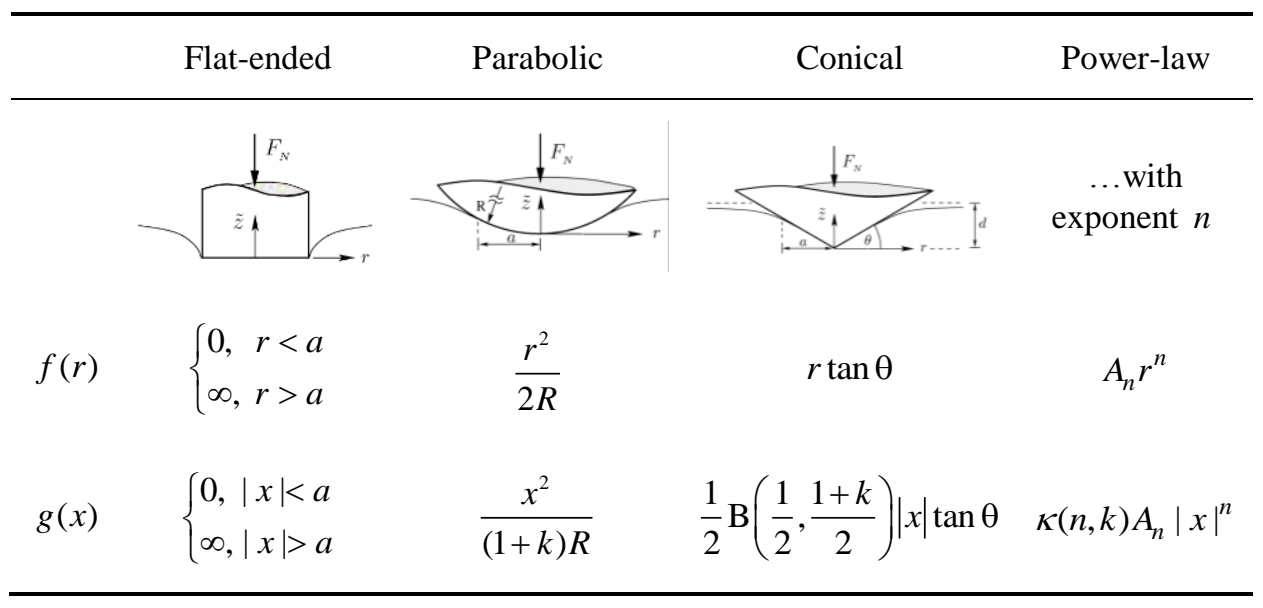

\section{CAlCUlation Rules of MDR FOR SOlVing NORMAl CONTACTS BETWEEN POWER-LAW GRADED MATERIALS WITHOUT ADHESION}

The MDR procedure for solving contact problems between power-law graded materials is the same as in the classical MDR for homogeneous materials. 


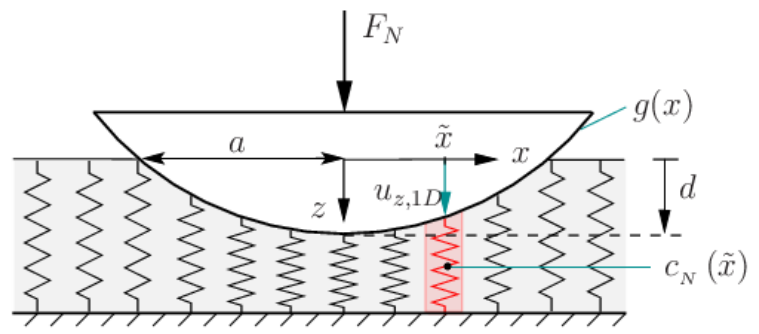

Fig. 5 Equivalent 1D contact problem of the 3D contact problem between two power-law graded half-spaces

The one-dimensional profile according to Eq. (5) is pressed into an elastic foundation of normal modulus given by Eq. (2) (see Fig. 5). The normal surface displacement at point $x$ within the contact area results from the difference between indentation depth $d$ and profile form $g$ :

$$
u_{z, 1 \mathrm{D}}(x):=d-g(x) \text {. }
$$

At the edge of non-adhesive contact $|x|=a$ the surface displacement must be zero:

$$
u_{z, 1 D}(a)=0 \Rightarrow d=g(a) \text {. }
$$

This equation determines the relationship between indentation depth $d$ and contact radius $a$. The sum of all spring forces must correspond to the normal force in equilibrium:

$$
F_{N}(a)=\int_{-a}^{a} c_{N}(x) u_{z, 1 D}(x) \mathrm{d} x=2 \int_{0}^{a} c_{N}(x)[d-g(x)] \mathrm{d} x .
$$

Eqs. (11) and (12) provide the penetration depth and the normal force as a function of the contact radius. The pressure distribution in the original three-dimensional system can be determined with the help of the one-dimensional displacement using the integral transformation:

$$
p(r, a)=-\frac{c_{N}\left(c_{0}\right)}{\pi c_{0}^{k}} \int_{r}^{\infty} \frac{u_{z, 1 D}^{\prime}(x)}{\left(x^{2}-r^{2}\right)^{\frac{1-k}{2}}} \mathrm{~d} x .
$$

The foundation modulus at position $c_{0}$ takes into account the elastic parameters of the elastically inhomogeneous materials in contact. From Eq. (2) follows:

$$
c_{N}\left(c_{0}\right)=\left(\frac{1-v_{1}^{2}}{h_{N}\left(k, v_{1}\right) E_{01}}+\frac{1-v_{2}^{2}}{h_{N}\left(k, v_{2}\right) E_{02}}\right)^{-1} .
$$

The normal surface displacement outside of the contact area is given by the transformation:

$$
u_{z}(r, a)=\frac{2 \cos \left(\frac{k \pi}{2}\right)}{\pi} \int_{0}^{a} \frac{x^{k} u_{z, 1 D}(x)}{\left(r^{2}-x^{2}\right)^{\frac{1+k}{2}}} \mathrm{~d} x \text { for } r>a
$$




\subsection{Examples for normal contacts without adhesion}

\subsubsection{Parabolic contact}

As the first example we consider a rigid, parabolic indenter, which is pressed into a power-law graded half-space. The shape function of the parabolic contact is defined by:

$$
f(r)=\frac{r^{2}}{2 R} .
$$

Using the transformation formula (5) yields the shape function of the equivalent onedimensional profile (see also Table 1):

$$
g(x)=\frac{x^{2}}{(k+1) R} .
$$

Thereby the displacement of the Winkler foundation is known, so that the indentation depth immediately emerges from Eq. (11):

$$
u_{z, 1 D}(a)=0 \Rightarrow d(a)=g(a)=\frac{a^{2}}{(k+1) R} .
$$

According to Eq. (12), the normal force results from the sum of the spring forces, taking into account the increasing foundation modulus from the center of the contact line according to Eq. (2):

$$
F_{N}(a)=\int_{-a}^{a} c_{N}(x)\left(\frac{a^{2}}{(1+k) R}-\frac{x^{2}}{(1+k) R}\right) d x=\frac{4 E_{0}}{\left(1-v^{2}\right)} \frac{h_{N}(k, v)}{c_{0}^{k}(k+1)^{2}(k+3)} \frac{a^{k+3}}{R} .
$$

To calculate the pressure distribution in the contact area, we need the first derivative of the 1D displacement:

$$
u_{z, 1 D}(x)=d-\frac{x^{2}}{(k+1) R} \Rightarrow u_{z, 1 D}^{\prime}(x)=-\frac{2 x}{(k+1) R}
$$

and the adjusted elastic parameter (one body was assumed to be rigid):

$$
c_{N}\left(c_{0}\right)=\frac{h_{N}(k, v) E_{0}}{1-v^{2}} .
$$

From Eq. (13) by taking Eqs. (20) and (21) into account, the pressure distribution is:

$$
p(r, a)=\frac{2 h_{N}(k, v) E_{0} a^{k+1}}{\pi\left(1-v^{2}\right) c_{0}^{k}(k+1)^{2} R}\left[1-\left(\frac{r}{a}\right)^{2}\right]^{\frac{1+k}{2}} .
$$

The application of Eq. (15) provides the normal displacement of the surface outside of the contact area: 


$$
u_{z}(r, a)=\frac{a^{2} \cos \left(\frac{k \pi}{2}\right)}{(k+1) \pi R}\left[\mathrm{~B}\left(\frac{a^{2}}{r^{2}}, \frac{1+k}{2}, \frac{1-k}{2}\right)-\frac{r^{2}}{a^{2}} \mathrm{~B}\left(\frac{a^{2}}{r^{2}}, \frac{3+k}{2}, \frac{1-k}{2}\right)\right] \text {, }
$$

with the incomplete beta function:

$$
\mathrm{B}(z, x, y):=\int_{0}^{z} t^{x-1}(1-t)^{y-1} \mathrm{~d} t \quad \forall x, y \in \mathbb{R}^{+} .
$$

It is easy to verify that from Eqs. (18), (19) as well as Eqs. (22), (23) in the particular case $k=0$ the solutions of the Hertzian contact exactly follow.

\subsubsection{Conical contact}

For the conical contact, the profile functions of the original and the equivalent system are listed in Table 1. They are:

$$
f(r)=r \tan \theta \Rightarrow g(x)=\frac{1}{2} \mathrm{~B}\left(\frac{1}{2}, \frac{1+k}{2}\right)|x| \tan \theta .
$$

Table 2 summarizes the solutions of the conical contact, which results from the MDR rules Eqs. (10)-(13) and (15). Again, one body was assumed to be rigid.

Table 2 Solutions to the normal contact between a rigid conical indenter and a powerlaw graded half-space

\begin{tabular}{c}
\hline$d(a) \quad \frac{1}{2} a \tan \theta \mathrm{B}\left(\frac{1}{2}, \frac{1+k}{2}\right)$ \\
$F_{N}(a) \quad \frac{h_{N}(k, v) \tan \theta \mathrm{B}\left(\frac{1}{2}, \frac{1+k}{2}\right) E_{0} a^{k+2}}{\left(1-v^{2}\right) c_{0}^{k}(k+1)(k+2)}$ \\
$\frac{h_{N}(k, v) \tan \theta \mathrm{B}\left(\frac{1}{2}, \frac{1+k}{2}\right) E_{0}}{4 \pi\left(1-v^{2}\right) c_{0}^{k}}\left[\mathrm{~B}\left(\frac{-k}{2}, \frac{1+k}{2}\right)-\mathrm{B}\left(\frac{r^{2}}{a^{2}}, \frac{-k}{2}, \frac{1+k}{2}\right)\right]$ \\
\hline$u_{z}(r, a) \quad \frac{\tan \theta \mathrm{B}\left(\frac{1}{2}, \frac{1+k}{2}\right) \cos \left(\frac{k \pi}{2}\right) a}{2 \pi}\left[\mathrm{B}\left(\frac{a^{2}}{r^{2}}, \frac{1+k}{2}, \frac{1-k}{2}\right)-\frac{r}{a} \mathrm{~B}\left(\frac{a^{2}}{r^{2}}, \frac{3+k}{2}, \frac{1-k}{2}\right)\right]$ \\
\hline
\end{tabular}


Since the beta function in the pressure distribution contains some negative arguments, we extend the definition according to Eq. (24), in which we make use of its representation by hypergeometric series:

$$
\mathrm{B}(z, x, y)=\frac{z^{x}}{x}{ }_{2} F_{1}(x, 1-y ; 1+x ; z)
$$

where

$$
{ }_{2} F_{1}(a, b ; c ; z)=\sum_{n=0}^{\infty} \frac{(a)_{n}(b)_{n}}{(c)_{n}} \frac{z^{n}}{n !} \quad \text { with } \quad(x)_{n}:=\frac{\Gamma(x+n)}{\Gamma(x)} .
$$

\section{AdHESIVE Normal CONTACT BETweEn POWER-LAw Graded Materials}

For the solution of the normal contact with adhesion between two power-law graded materials by means of MDR, there is only a small change to the non-adhesive normal contact: the rule according to Eq. (11) for calculating the indentation depth as a function of the contact radius must simply be replaced by rule [14]:

$$
u_{z, 1 D}(a)=-\Delta \ell_{\max }(a) \text { with } \Delta \ell_{\max }(a):=\sqrt{\frac{2 \pi \Delta \gamma a}{c_{N}(a)}} .
$$

Clearly, this means that the equilibrium state of the contact with adhesion is found when the elongations of the springs at the edge of contact reach defined value $\Delta \ell_{\max }(a)$ (see Fig. 6).

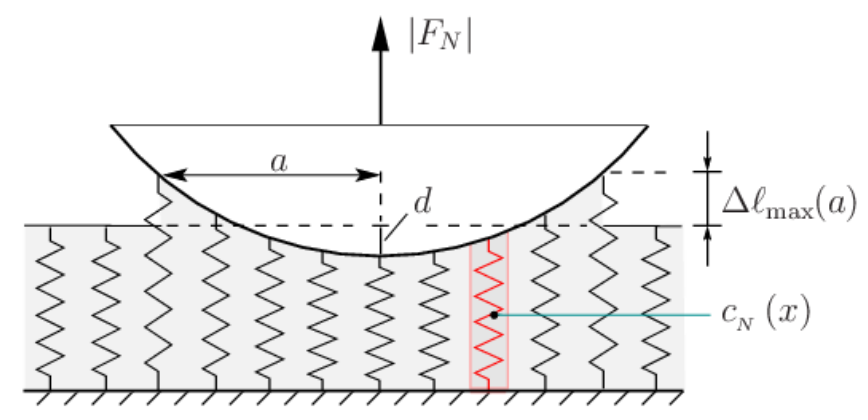

Fig. 6 Illustration of the MDR rule for an adhesive contact between power-law graded materials

In addition, the MDR solution provides a simple way to calculate the critical contact radii, and thus the (maximum) pull-off force as well as the minimum indentation depth The critical contact radii must fulfill the following condition: 


$$
\frac{\Delta \ell_{\max }\left(a_{c}\right)}{a_{c}}=\left.\tilde{C}(k) \frac{\partial g(a)}{\partial a}\right|_{a_{c}} \text { with } \tilde{C}(k):= \begin{cases}\frac{2}{3+k} & \text { for } \text { fixed-load } \\ \frac{2}{1-k} & \text { for } \text { fixed-grips }\end{cases}
$$

Thus, the slope of the equivalent profile at the contact edge is decisive for reaching the critical states. The different definition of coefficient $\tilde{C}(k)$ is linked to whether a fixedload or fixed-grips condition is present. With conditions (27), (28) and the general rules of the MDR procedure (10), (12), (13) and (15), every standard contact problem with adhesion can be easily solved. A few examples are presented below.

\subsection{Examples for adhesive normal contacts}

\subsubsection{Parabolic contact}

We refrain from re-calculating the equivalent profile for the parabolic contact at this point and adopt the result of Eq. (17):

$$
f(r)=\frac{r^{2}}{2 R} \Rightarrow g(x)=\frac{x^{2}}{(k+1) R} .
$$

Considering the definition of the displacement of the Winkler foundation from Eq. (10), the indentation depth as a function of the contact radius for the adhesive contact follows from the separation criterion (27):

$$
d(a)=\frac{a^{2}}{(k+1) R}-\sqrt{\frac{2 \pi \Delta \gamma c_{0}^{k} a^{1-k}}{E^{*} h_{N}(k, v)}},
$$

where we abbreviated $E^{*}:=E_{0} /\left(1-v^{2}\right)$. According to Eq. (12), the normal force must again correspond to the sum of all spring forces. It differs from the normal force in the case of the non-adhesive contact only by a part which results from an additional rigid-body translation $-\Delta \ell_{\max }(a)$ of all springs:

$$
\begin{aligned}
F_{N}(a) & =\frac{2}{(1+k) R} \int_{0}^{a} c_{N}(x)\left(a^{2}-x^{2}\right) d x-2 \int_{0}^{a} c_{N}(x) \Delta \ell_{\max }(a) d x \\
& =\frac{4 h_{N}(k, v) E^{*} a^{k+3}}{c_{0}^{k}(k+1)^{2}(k+3) R}-\sqrt{\frac{8 \pi \Delta \gamma h_{N}(k, v) E^{*} a^{3+k}}{(k+1)^{2} c_{0}^{k}}}
\end{aligned}
$$

For the calculation of the critical contact radii from condition (28) (limit stability), only the slope of the equivalent profile at the contact edge is required. From Eq. (29) it follows $g^{\prime}(a)=2 a /[(1+k) R]$ and with it from Eq. (28):

$$
a_{c}=\left(\frac{\pi(1+k)^{2} R^{2} \Delta \gamma c_{0}{ }^{k}}{2 \tilde{C}(k)^{2} h_{N}(k, v) E^{*}}\right)^{\frac{1}{3+k}} .
$$


Inserting the critical contact radii according to Eq. (32) into Eqs. (30) and (31) provides the critical indentation depths and normal forces:

$$
\begin{gathered}
d_{c}=\frac{1-2 \tilde{C}(k)}{(1+k) R}\left(\frac{\pi(1+k)^{2} R^{2} \Delta \gamma c_{0}^{k}}{2 \tilde{C}(k)^{2} h_{N}(k, v) E^{*}}\right)^{\frac{2}{3+k}}, \\
F_{c}=\left(\frac{1-\tilde{C}(k)(3+k)}{\tilde{C}(k)^{2}(3+k)}\right) 2 \pi \Delta \gamma R=\left\{\begin{array}{ll}
-\frac{k+3}{2} \pi \Delta \gamma R & \text { (fixed-load) } \\
-\frac{(1-k)(5+3 k)}{2(3+k)} \pi \Delta \gamma R & \text { (fixed-grips) }
\end{array} .\right.
\end{gathered}
$$

It is needless to say that Eqs. (30)-(34) developed by MDR agree exactly with solutions from three-dimensional theory by Chen et al. [7]. They drew attention to the fact that, according to Eq. (34), the maximum pull-off force is independent of the elastic parameters and independent of the characteristic depth as in the homogeneous case. For the calculation of the pressure distribution according to Eq. (13), we need the one-dimensional displacement respectively its derivative, which we specify here again to clarify the treatment of the finite jump at the contact edge (see Fig. 6):

$$
\begin{gathered}
u_{z, 1 \mathrm{D}}(x)=\left(\frac{a^{2}-x^{2}}{(k+1) R}-\Delta \ell_{\text {max }}(a)\right)[\mathrm{H}(x+a)-\mathrm{H}(x-a)] \text { with } x \in \mathbb{R}, \\
u_{z, 1 \mathrm{D}}^{\prime}(x)=-\frac{2 x}{(k+1) R}[\mathrm{H}(x+a)-\mathrm{H}(x-a)] \ldots \\
\ldots+\left(\frac{a^{2}-x^{2}}{(k+1) R}-\Delta \ell_{\text {max }}(a)\right)[\delta(x+a)-\delta(x-a)]
\end{gathered}
$$

In Eqs. (35) and (36) $\mathrm{H}(\ldots)$ denote the Heaviside function and $\delta(\ldots)$ the delta distribution. After insertion of Eq. (36) in Eq. (13) and taking into account the filter property of the delta distribution, the pressure distribution results in:

$$
p(r, a)=\frac{2 h_{N}(k, v) E^{*} a^{k+1}}{\pi c_{0}^{k}(k+1)^{2} R}\left[1-\left(\frac{r}{a}\right)^{2}\right]^{\frac{1+k}{2}}-\sqrt{\frac{2 h_{N}(k, v) \Delta \gamma E^{*} a^{k-1}}{\pi c_{0}{ }^{k}}}\left[1-\left(\frac{r}{a}\right)^{2}\right]^{\frac{k-1}{2}} .
$$

\subsubsection{Power-law contact profile}

The equivalent profile of an indenter whose shape is a power function according to Eq. (7) has already been calculated in Eq. (8):

$$
f(r)=A_{n} r^{n} \Rightarrow g(x)=\kappa(n, k) A_{n}|x|^{n} \quad, n \in \mathbb{R}^{+}
$$

wherein $\kappa(n, k)$ has been defined in Eq. (9). The solutions of the adhesive contact between a power-law graded half-space and a rigid indenter whose profile is given by Eq. (38) using MDR are summarized in Table 3. 
Table 3 Solutions to the adhesive contact between a rigid indenter whose shape is a power function and a power-law graded half-space

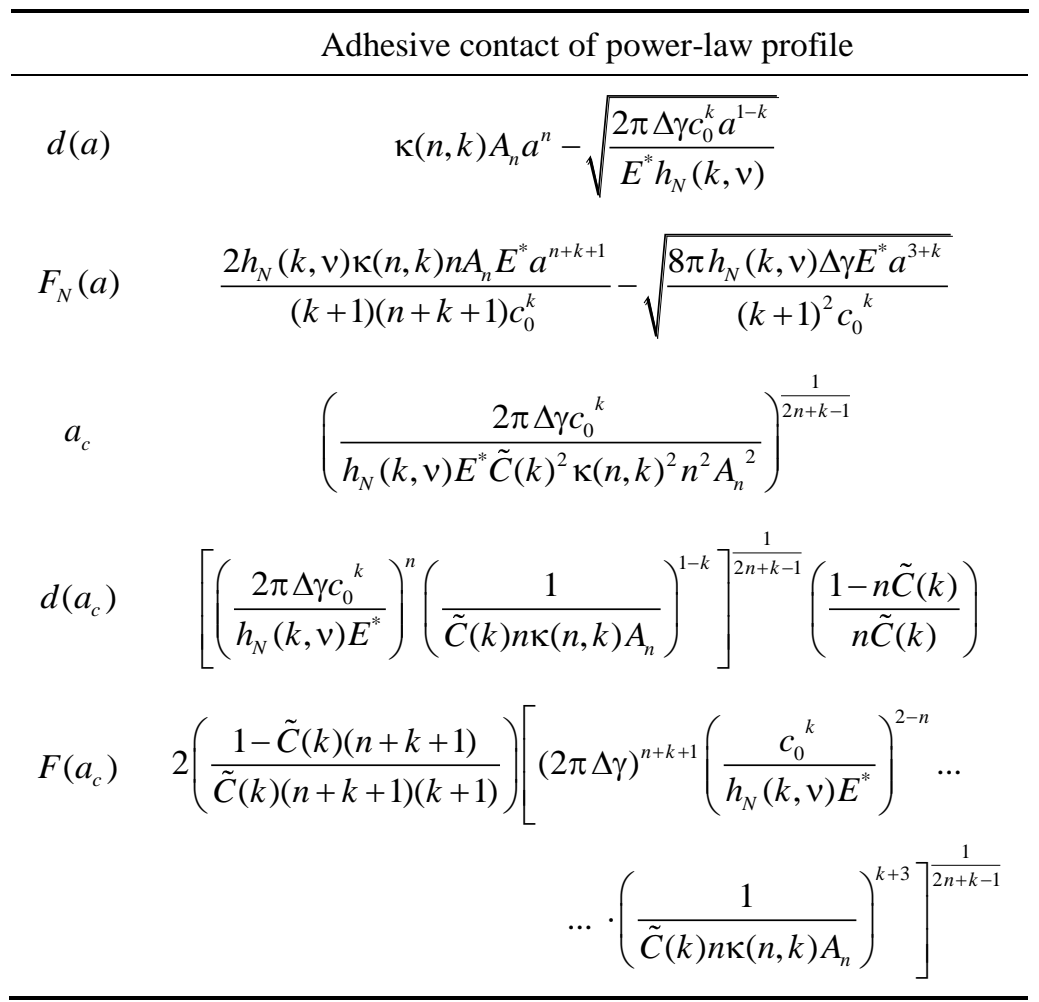

\section{TANGential Contact Between Two Power-Law Graded HalF-SpaCes}

We now consider a partial-slip problem between two power-law graded half-spaces with the same exponent $k$ of elastic inhomogeneity, but different elastic parameters $E_{0}$ and $v$. The solids are initially pressed against each other with a normal force $F_{N}$ and subsequently loaded with a tangential force $F_{x}$ in the $x$-direction (see Fig. 7). The axisymmetric gap function is given by $f(r)$. Let us assume that normal and tangential contacts are uncoupled, which is strictly permitted only if either [9]:

- both materials are equal: $v_{1}=v_{2}=: \vee \wedge E_{01}=E_{02}=: E_{0}$,

- one material is rigid and the other one has a Poisson's ratio equal to the Holl-ratio [16]: $E_{0 i} \rightarrow \infty \wedge v_{j}=1 /(2+k)$ with $i \neq j$ or

- both materials have a Poisson's ratio which corresponds to the Holl-ratio: $v_{1}=v_{2}=1 /(2+k)$. 


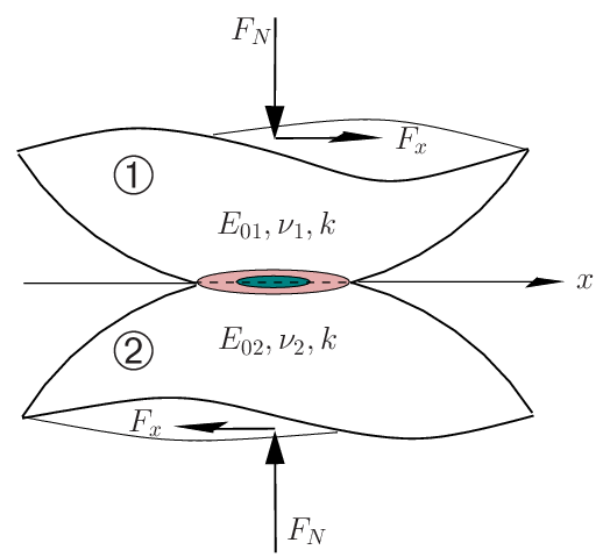

Fig. 7 Tangential contact between two power-law graded half-spaces

It is well-known that the contact region consists of an inner stick and outer slip region. In the stick domain all points undergo the same tangential displacement $\delta_{x}$. The (undirectional assumed) tangential stresses are determined by Coulomb's law of friction:

$$
\begin{gathered}
|\tau(r)| \leq \mu p(r) \quad \text { for } \quad(x, y) \in A_{\text {stick }}, \\
|\tau(r)|=\mu p(r) \quad \text { for } \quad(x, y) \in A \backslash A_{\text {stick }} .
\end{gathered}
$$

We denote the radius of the stick domain by $c$.

The equivalent model for the tangential contact is shown in Fig. 8. As already mentioned, each spring has normal and tangential stiffness according to Eqs. (2) and (3). We note once again that these stiffnesses depend on the lateral coordinate according to a power law.
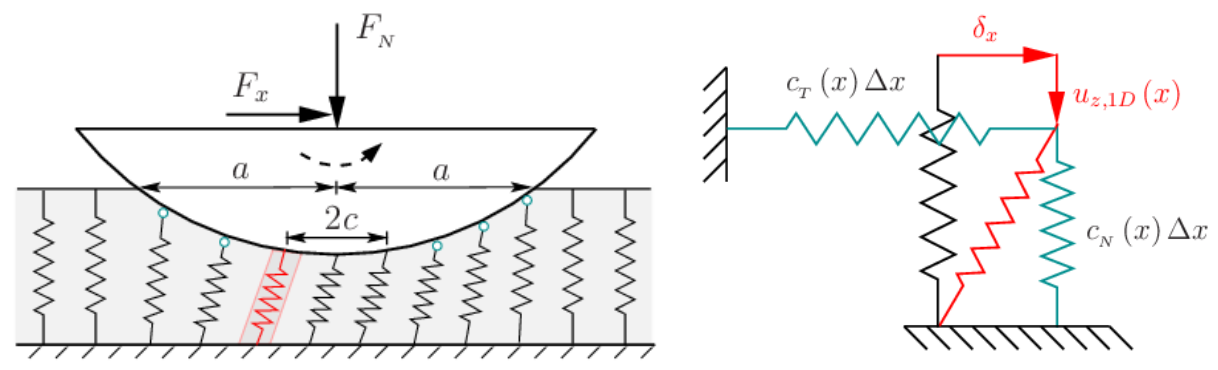

Fig. 8 Equivalent model for the partial-slip problem between two power-law graded half-spaces; each spring has normal and tangential stiffness, which are independent of each other

Due to the uncoupled normal and tangential contact, we assume the solution of the normal contact problem according to Section 3 as already known. The MDR-rules for the solution of the tangential contact require Amonton's law for each spring. The tangential line load is thus defined as follows: 


$$
q_{x}(x)=\left\{\begin{array}{cccc}
c_{T}(x) \delta_{x} & \text { for } & |x| \leq c \quad \text { (stick) } \\
\mu \underbrace{c_{N}(x) u_{z, 1 D}(x)}_{=q_{z}(x)} & \text { for } & c<|x| \leq a \text { (slip) . }
\end{array}\right.
$$

It takes into account both the rigid-body translation of the stick area and the Coulomb friction in the sliding area. The calculation of the stick radius is based on the continuity of the tangential line load at the transition between stick and slip domain:

$$
\lim _{x \rightarrow c^{-}} q_{x}(x)=\lim _{x \rightarrow c^{+}} q_{x}(x)=q_{x}(c) \Rightarrow \delta_{x}=\mu \frac{c_{N}(c)}{c_{T}(c)} u_{z, 1 D}(c) .
$$

Analogously to the normal contact problem, the tangential force results from the sum of the tangential spring forces. If, instead of the spring forces, the line load from Eq. (41) is used, the calculation formula is:

$$
F_{x}(a)=\int_{-a}^{a} q_{x}(x) d x=2 \int_{0}^{c} c_{T}(x) \delta_{x} d x+2 \int_{c}^{a} \mu c_{N}(x) u_{z, 1 D}(x) d x
$$

It is also possible to deduce the pressure distribution of the original contact problem from the equivalent model. For this purpose, the knowledge of the tangential line load is sufficient since:

$$
\tau(r):=-\tau_{z x}(r)=-\frac{1}{\pi r} \frac{\mathrm{d}}{\mathrm{d} r} \int_{r}^{\infty} \frac{x^{1-k} q_{x}(x)}{\left(x^{2}-r^{2}\right)^{\frac{1-k}{2}}} \mathrm{~d} x .
$$

It should be noted that the tangential line load according to Eq. (41) can also be expressed as a difference of the vertical line loads:

$$
q_{x}(x)=\mu\left[q_{z}(x, a) \cdot \mathrm{H}(a-|x|)-q_{z}(x, c) \cdot \mathrm{H}(c-|x|)\right] .
$$

where $q_{z}(x, a)$ is the normal line load actually acting in the equivalent model, and $q_{z}(x, c)$ is one that belongs to a smaller contact radius, stick radius $c$.

\subsection{Example: Parabolic tangential contact between power-law graded half-spaces}

In the following the same power-law graded materials are assumed which allow uncoupling of normal and tangential contact. The normal contact problem has already been described by means of the MDR in the examples of Section 3. The only difference is that one solid is assumed to be rigid. In order to be able to adopt the solution, we only have to adjust the stiffness, which is half as large, since both bodies are elastic. Regardless of it, the stiffness is shown here again:

$$
\begin{aligned}
& c_{N}(x)=\frac{h_{N}(k, v) E_{0}}{2\left(1-v^{2}\right)}\left(\frac{|x|}{c_{0}}\right)^{k}, \\
& c_{T}(x)=\frac{1}{2} h_{T}(k, v) E_{0}\left(\frac{|x|}{c_{0}}\right)^{k} .
\end{aligned}
$$


The one-dimensional normal displacement of the Winkler foundation has already been determined (see Eqs. (20), (18)) so that the tangential line load can be specified:

$$
q_{x}(x)=\left\{\begin{array}{clll}
c_{T}(x) \delta_{x} & \text { for } & |x| \leq c \quad \text { (stick) } \\
\mu c_{N}(x) \frac{a^{2}-x^{2}}{(1+k) R} & \text { for } & c<|x| \leq a \text { (slip) }
\end{array} .\right.
$$

From the continuity requirement Eq. (42) at the points $|x|=c$ of the tangential line load it follows:

$$
\delta_{x}=\mu \frac{h_{N}(k, v) a^{2}}{\left(1-v^{2}\right) h_{T}(k, v)(1+k) R}\left(1-\frac{c^{2}}{a^{2}}\right) .
$$

The maximum displacement before macroscopic sliding (full slip) begins is thus:

$$
\delta_{x, \max }=\frac{\mu h_{N}(k, v) a^{2}}{\left(1-v^{2}\right) h_{T}(k, v)(1+k) R} .
$$

Integration of the tangential line load (48) over contact length $2 a$ according to Eq. (43) yields:

$$
\frac{F_{x}}{\mu F_{N}}=1-\left(\frac{c}{a}\right)^{k+3}=1-\left(1-\frac{\delta_{x}}{\delta_{x, \max }}\right)^{\frac{k+3}{2}}
$$

where we have taken into account Eqs. (49) and (50) on the right.

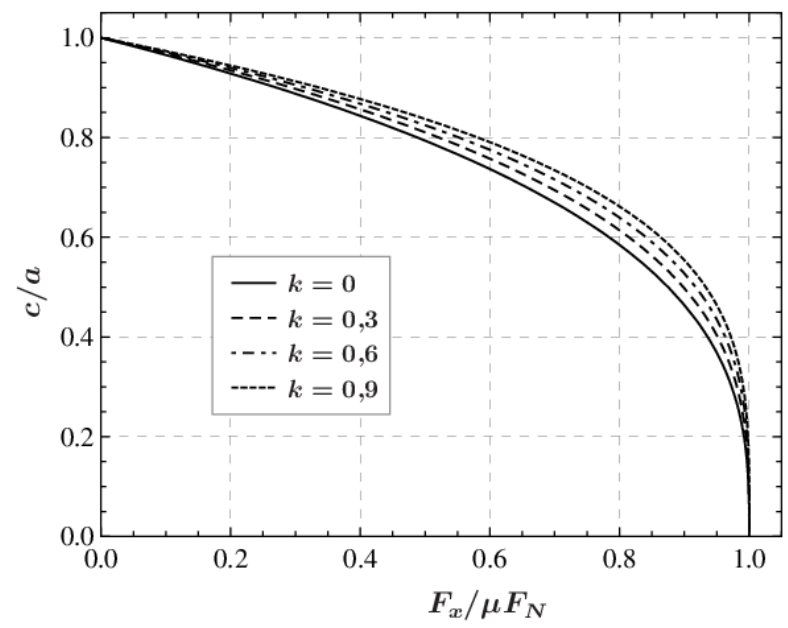

Fig. 9 Stick radius as a function of tangential force in normalized representation for different exponents of elastic inhomogeneity $k$

Fig. 9 shows the dependence of the stick radius on the tangential force for different exponents $k$. The tangential force as a function of the tangential displacement for the spe- 
cial case $v_{1}=v_{2}=1 /(2+k)$ is depicted in Fig. 10. In this case, the prefactor in the maximum displacement deflates and from Eq. (50) it follows:

$$
\delta_{x, \max }=\mu \frac{3+k}{2} \frac{a^{2}}{R} .
$$

In Fig. 10 the tangential shift was normalized to the maximum tangential displacement in the homogeneous case. As can be seen from Eq. (52), the tangential shift increases with increasing $k$. When the state of full-slip is reached, a 30\% larger displacement is obtained for $k=0.9$ in comparison to the homogeneous case.

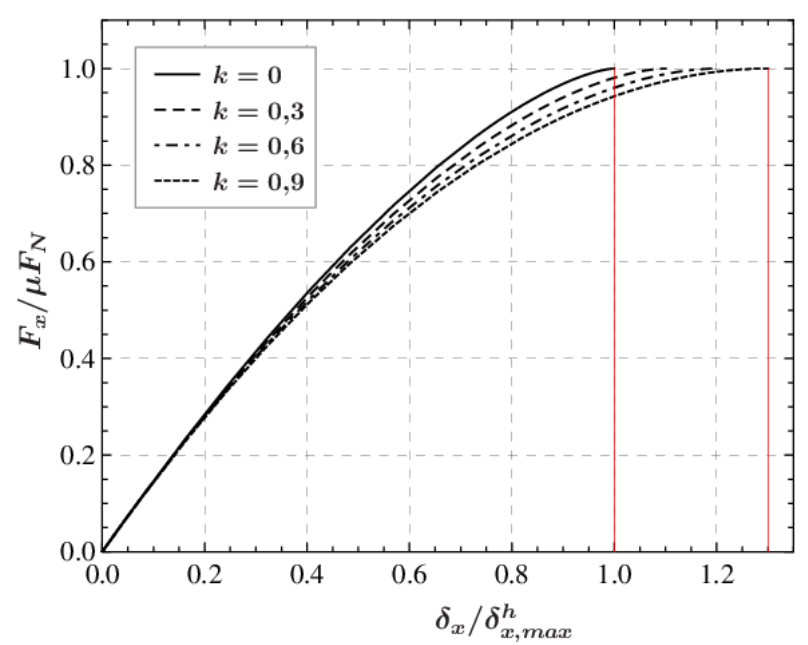

Fig. 10 Normalized representation of the dependence between tangential force and tangential displacement for different exponents of elastic inhomogeneity and specification of $v_{1}=v_{2}=1 /(2+k)$

Finally, it should be noted that we have assumed the usual approximations which are already contained in the classic solution of Cattaneo [17] and Mindlin [18] and have been discussed in detail by Ciavarella [11]. With reference to the corresponding paper we therefore waive an explicit listing.

\section{CONCLUSIONS}

This paper presents all the essential rules of the MDR that allow the solution of contact problems between power-law graded materials. We have carefully distinguished between normal, tangential and adhesive contacts and explained the simple application of the rules by means of examples. It does not need to be mentioned that despite its simplicity the MDR reproduces exactly all the results of the complicated three-dimensional theory. We would like to emphasize that the analytical solution of the tangential contact between power-law graded materials is an absolute novelty, since a derivation from the three-dimensional theory was missing so far. It was only in the run-up to the present pub- 
lication that this gap could be closed [9]. Clearly, all MDR rules for solving contact problems between elastically homogenous materials are a special case of the rules presented here. The presented extension of the MDR is of interest to a number of current research areas since functionally-graded materials are gaining in importance. These include tribology, nanotechnology, biostructure mechanics and medicine. Completely analogous to the calculation of wear profiles between homogeneous materials $[19,20]$, the investigation of fretting between elastically inhomogeneous materials should no longer constitute a barrier.

The same applies to the extension of the current numerically simulated impact problems between elastically homogeneous spheres [21, 22] onto elastically inhomogeneous ones. Based on the MDR-rules presented here, which are comprehensible to everyone, the development of asymptotic solutions [23] for complicated contact configurations between powerlaw graded materials is also likely to be much easier.

We would like to point out that the theory presented here is limited to power-law graded materials. The extent to which the theory can be applied to other laws of elastic inhomogeneity remains a challenging, future task.

\section{APPENDIX}

The coefficients contained in the foundation moduli according to Eqs. (2), (3) are defined as follows $[9,15]$ :

$$
\begin{gathered}
h_{N}(k, v)=\frac{2(1+k) \cos \left(\frac{k \pi}{2}\right) \Gamma\left(1+\frac{k}{2}\right)}{\sqrt{\pi} C(k, v) \beta(k, v) \sin \left(\frac{\beta(k, v) \pi}{2}\right) \Gamma\left(\frac{1+k}{2}\right)}, \\
h_{T}(k, v)=\frac{4 \beta(k, v) \cos \left(\frac{k \pi}{2}\right) \Gamma\left(1+\frac{k}{2}\right)}{\left(1-v^{2}\right)(1+k) \sqrt{\pi} C(k, v) \sin \left(\frac{\beta(k, v) \pi}{2}\right) \Gamma\left(\frac{1+k}{2}\right)+2 \beta(k, v)(1+v) \Gamma\left(1+\frac{k}{2}\right)}
\end{gathered}
$$

with

$$
C(k, v)=\frac{2^{1+k} \Gamma\left(\frac{3+k+\beta(k, v)}{2}\right) \Gamma\left(\frac{3+k-\beta(k, v)}{2}\right)}{\pi \Gamma(2+k)}
$$

and

$$
\beta(k, v)=\sqrt{(1+k)\left(1-\frac{k v}{1-v}\right)} .
$$




\section{REFERENCES}

1. Popov, V.L. and Hess, M., 2015, Method of dimensionality reduction in contact mechanics and friction. Berlin Heidelberg: Springer-Verlag

2. Popov, V.L. and Hess, M., 2014, Method of dimensionality reduction in contact mechanics and friction: a users handbook. I. Axially-symmetric contacts, Facta Universitatis, Series: Mechanical Engineering, 12(1), pp. 1-14.

3. Argatov, I., Hess, M., Pohrt, R., Popov, V.L., 2016, The extension of the method of dimensionality reduction to non-compact and non-axisymmetric contacts. ZAMM-Journal of Applied Mathematics and Mechanics, 96(10), pp. 1144-1155, doi:10.1002/zamm.201600057

4. Booker, J.R., Balaam, N.P., Davis, E.H., 1985, The behaviour of an elastic non-homogeneous half-space. Part I-line and point loads, International Journal for Numerical and Analytical Methods in Geomechanics, 9(4), pp. 353-367.

5. Booker, J.R., Balaam, N.P., Davis, E.H., 1985, The behaviour of an elastic non-homogeneous half-space. Part II-circular and strip footings, International journal for numerical and analytical methods in geomechanics, 9(4), pp. 369-381.

6. Giannakopoulos, A. E., Suresh, S., 1997, Indentation of solids with gradients in elastic properties: Part II, Axisymmetric indentors, International Journal of Solids and Structures, 34(19), pp. 2393-2428.

7. Chen, S., Yan, C., Zhang, P., Gao, H, 2009, Mechanics of adhesive contact on a power-law graded elastic half-space, Journal of the Mechanics and Physics of Solids, 57(9), pp. 1437-1448.

8. Jin, F., Guo, X. and Zhang, W., 2013, A unified treatment of axisymmetric adhesive contact on a powerlaw graded elastic half-space, Journal of Applied Mechanics, 80(6), p. 061024.

9. Hess, M., 2016, Normal, tangential and adhesive contacts between power-law graded materials, Presentation at the Workshop on Tribology and Contact Mechanics in Biological and Medical Applications, TU-Berlin, 14.17. Nov. 2016

10. Hess, M., Popov, V.L., 2016, Die Renaissance der Winklerschen Bettung in der Kontaktmechanik und Reibungsphysik - Eine Anwendung auf Kontaktprobleme funktioneller Gradientenwerkstoffe, Conference Paper, Tribologie-Fachtagung, 04, pp. 1-11

11. Ciavarella M., 1998, Tangential Loading of General Three-Dimensional Contacts. Journal of Applied Mechanics, 65, pp. 998-1003.

12. Jaeger, J., 1995, Axi-symmetric bodies of equal material in contact under torsion or shift, Archive of Applied Mechanics, 65, pp. 478-487.

13. Popov, V.L., 2014, Method of dimensionality reduction in contact mechanics and tribology. Heterogeneous media, Physical Mesomechanics, 17(1), pp. 50-57.

14. Hess, M., 2016, A simple but precise method for solving axisymmetric contact problems involving elastically graded materials, arXiv preprint arXiv:1602.04720.

15. Hess, M., 2016, A simple method for solving adhesive and non-adhesive axisymmetric contact problems of elastically graded materials, International Journal of Engineering Science, 104, pp. 20-33.

16. Holl, D.L., 1940, Stress transmission in earths, Highway Research Board Proceedings, 20, pp. 709-721.

17. Cattaneo, C., 1938, Sul contatto di due corpi elastici: distribuzione locale degli sforzi, Rendiconti dell'Accademia nazionale dei Lincei, 27, pp. 342-348, 434-436, 474-478.

18. Mindlin, R.D., 1949, Compliance of elastic bodies in contact, Journal of Applied Mechanics, 16(3), pp. 259-268.

19. Popov, V.L., 2014, Analytic solution for the limiting shape of profiles due to fretting wear, Sci. Rep., 4, 3749.

20. Li, Q., 2016, Limiting profile of axisymmetric indenter due to the initially displaced dual-motion fretting wear, Facta Universitatis, Series: Mechanical Engineering, 14(1), pp. 55-61.

21. Lyashenko, I.A., Willert, E., Popov, V.L., 2016, Adhesive impact of an elastic sphere with an elastic half space: Numerical analysis based on the method of dimensionality reduction, Mechanics of Materials, 92, pp. 155-163.

22. Willert, E., Popov, V.L., 2016, Impact of an elastic sphere with an elastic half space with a constant coefficient of friction: Numerical analysis based on the method of dimensionality reduction, ZAMM-Journal of Applied Mathematics and Mechanics, 96(9), pp. 1089-1095, DOI: 10.1002/zamm.201400309

23. Argatov, I., Li, Q., Pohrt, R., Popov, V.L., 2016, Johnson-Kendall-Roberts adhesive contact for a toroidal indenter, Proc. R. Soc: A, 472(2919): 20160218 